\title{
Quantification of the troposphere-to-ionosphere charge transfer in a gigantic jet
}

\author{
Steven A. Cummer ${ }^{1 \star}$, Jingbo Li ${ }^{1}$, Feng Han ${ }^{1}$, Gaopeng Lu', Nicolas Jaugey ${ }^{1}$, Walter A. Lyons ${ }^{2}$ \\ and Thomas E. Nelson ${ }^{2}$
}

Gigantic jets are the clearest manifestation of direct electrical coupling between tropospheric thunderstorms and the ionosphere. They are leaders ${ }^{1-3}$ that emerge from electrical breakdown near the top of thunderstorms ${ }^{4}$ and extend all the way to the lower edge of the ionosphere near $90 \mathrm{~km}$ altitude ${ }^{5}$. By contrast, blue jets ${ }^{6}$ and other related events ${ }^{7,8}$ terminate at much lower altitudes. Gigantic jets have been observed from the ground ${ }^{5,9,10}$ and from orbit ${ }^{11}$. Some seem to be consistent with an upward-propagating negative discharge of 1,000 to $2,000 \mathrm{C} \mathrm{km}$ total charge moment change ${ }^{9}$, but others have not been connected to distinguishable electromagnetic signatures ${ }^{10}$. Here we report simultaneous low-light video images and low-frequency magnetic field measurements of a gigantic jet that demonstrate the presence and dynamics of a substantial electric charge transfer between the troposphere and the ionosphere. The signatures presented here confirm the negative polarity of gigantic jets ${ }^{4}$ and constrain the lightning processes associated with them. The observed total charge transfer from the thunderstorm to the ionosphere is $144 \mathrm{C}$ for the assumed channel length of $\mathbf{7 5} \mathbf{~ k m}$, which is comparable to the charge transfer in strong cloud-to-ground lightning strokes.

At a field site near Duke University, we routinely monitor optical emissions above thunderstorms and measure $<0.1 \mathrm{~Hz}-30 \mathrm{kHz}$ magnetic field emissions from lightning and related processes. The radio signals provide a detailed view into the electric current and charge motion associated with the phenomena captured on triggered video. On 21 July 2008, a video sequence of high-altitude optical emissions above Tropical Storm Cristobal was captured. The inset of Fig. 1a shows the brightest image of the event at 03:10:53.229 UT (UT: universal time). The full image sequence (see Fig. 3) shows features that almost certainly classify this event as a gigantic jet ${ }^{9}$, namely the trailing jet feature and the long duration of continuous optical emissions (>350 ms).

We estimate a $350 \mathrm{~km}$ range to this event and a maximum jet altitude of $88 \mathrm{~km}$, which is consistent with other reports of gigantic jet altitudes ${ }^{5,9,10}$. The inferred geographic location of the jet is $33.63^{\circ} \mathrm{N}, 76.51^{\circ} \mathrm{W}$. When overlaid on a GOES-12 (GOES: Geostationary Operational Environmental Satellite) infrared image of the storm acquired $10 \mathrm{~min}$ before the jet event (Fig. 1a), the jet is seen to have occurred in the outer bands of the storm in the coldest region outside the storm core, with $-64{ }^{\circ} \mathrm{C}$ cloud tops corresponding to $14.0-14.5 \mathrm{~km}$ altitude (according to local soundings) over a roughly $50 \mathrm{~km}$ diameter region. A connection between high clouds and gigantic jets has been suggested ${ }^{4}$.

Radar reflectivity measured two minutes before the event (Fig. 1b) shows that the geographically estimated jet location is within a few kilometres of a strong core with peak base reflectivity

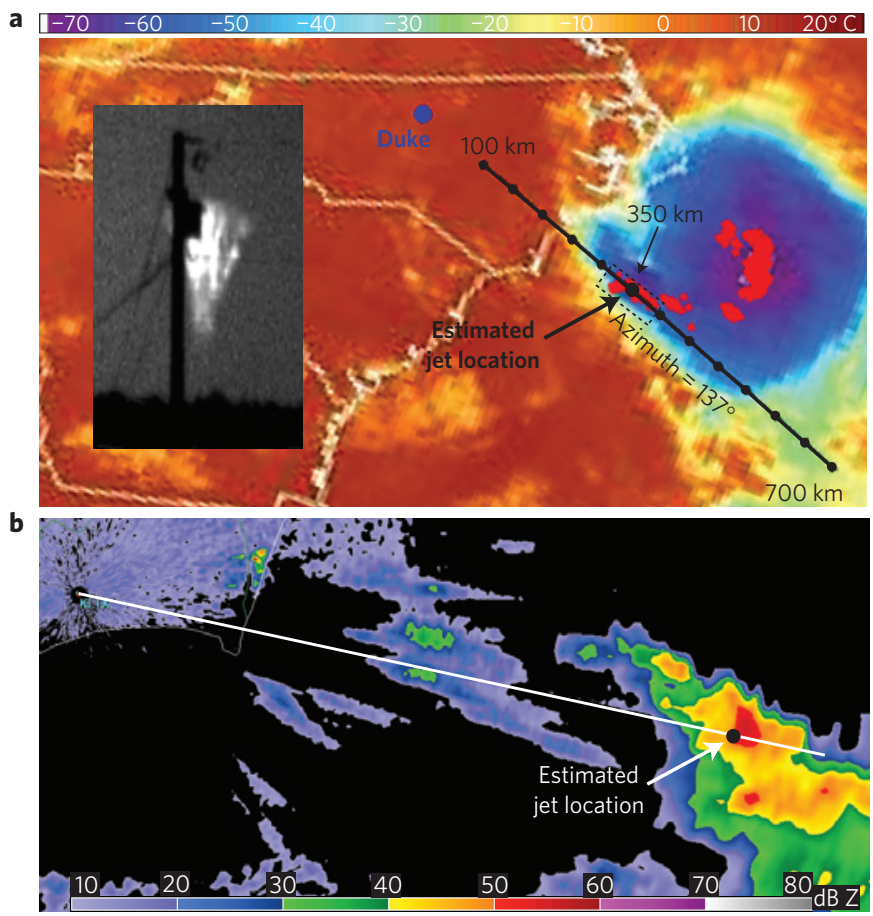

Figure 1 | Overview of the gigantic jet. a, NLDN-detected lightning strokes (red dots) from $\pm 10 \mathrm{~min}$ of the gigantic jet overlaid on a satellite infrared (temperature) image of the storm taken 10 min before the jet occurred. Also shown is the estimated jet location computed from the azimuth and range of the event. Inset: The brightest video frame from the captured sequence. $\mathbf{b}$, Radar reflectivity measured two minutes before the gigantic jet occurred, from Wilmington, North Carolina, relative to the estimated jet location.

just over $50 \mathrm{dBZ}$. The echo top map (not shown) shows $15.3 \mathrm{~km}$ echo tops in this deep, penetrative core, with cloud tops somewhat higher. Collectively these data suggest that the jet was associated with this intense, $10-\mathrm{km}$-wide overshooting convective dome that penetrated the general infrared-observed cloud canopy by $\sim 1 \mathrm{~km}$.

On the satellite image, the locations of all 981 lightning strokes detected by the National Lightning Detection Network (NLDN) that occurred in the storm $\pm 10 \mathrm{~min}$ from the jet time show that the jet occurred over an electrically active part of the storm. A total of 91 strokes were detected in this time window in the immediate vicinity of the gigantic jet (marked by the dotted rectangle in Fig. 1a), of which 77 were classified as negative cloud-to-ground 

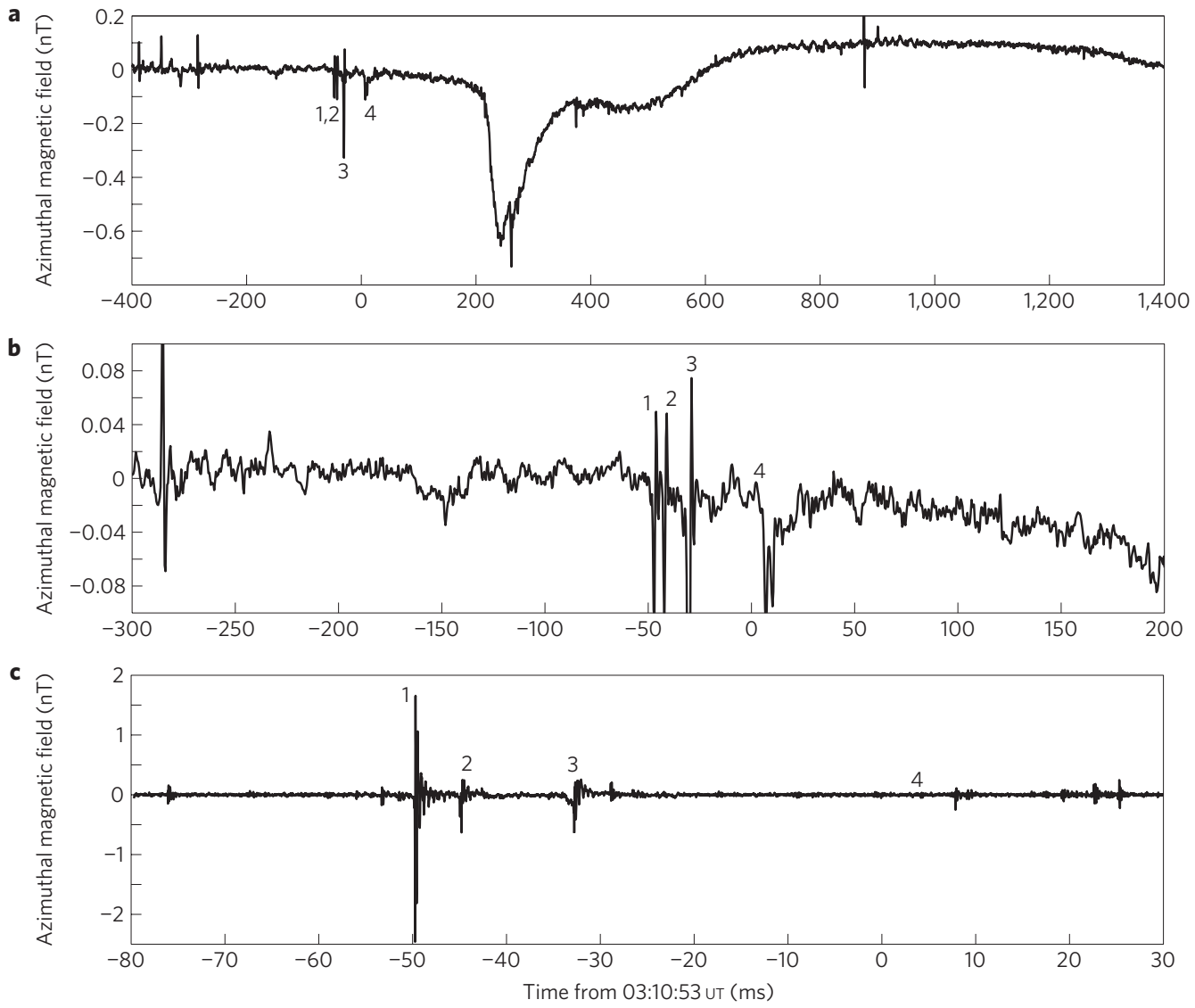

Figure 2 | VLF and ULF azimuthal magnetic fields produced by the gigantic jet. a, A $1.8 \mathrm{~s}$ window of the continuous ULF data showing a nearly $1 \mathrm{nT}$ pulse with an unusually slow rise and fall time. b. A close-up of the ULF data showing four distinct, short, and small pulses that occur around the time at which the slow rise begins. c, Simultaneously triggered VLF data that show the same four pulses as occurred near the onset of the gigantic jet.

and 5 as positive cloud-to-ground. The NLDN-reported lightning rate in this region was steady over this 20 min period, with no clear increase or decrease before or after the jet. No NLDN-detected strokes occurred within the entire duration of the jet, although the NLDN detection efficiency is not 100\% (ref. 12).

We use continuous $0.1-400 \mathrm{~Hz}$ (down to ultralow-frequency or ULF) and triggered $50 \mathrm{~Hz}-30 \mathrm{kHz}$ (up to very low-frequency or VLF) magnetic field recordings to determine what lightning processes occurred before or during the jet and whether the gigantic jet itself contains detectable electric current. Figure 2a shows the azimuthal component (assuming a source at the jet location) of the ULF magnetic field during the gigantic jet event. We use the time relative to $03: 10: 53.000$ uT to index the event as this reference seems to be within $50 \mathrm{~ms}$ of its beginning. The brightest jet image overlaps the peak of the ULF signature at $t=240 \mathrm{~ms}$ and the arrival direction of the ULF signal is the same as that of the optical event, linking the optical emissions and magnetic fields with none of the timing ambiguity or uncertainty seen in previous work ${ }^{9,10}$. The main pulse starting at $t=200 \mathrm{~ms}$ shows a slow, $30 \mathrm{~ms}$ rise time that clearly distinguishes it from a normal lightning return stroke, even those unusual strokes that create sprites $^{13}$. The positive overshoot of this pulse after $t=600 \mathrm{~ms}$ reflects the frequency response of the sensor and not a polarity reversal in the source current.

This signature indicates significant vertical current flow and thus charge transfer during the event. The source altitude cannot be distinguished from these data, however, as at these low frequencies a source in the Earth-ionosphere waveguide generates fields almost independent of source altitude ${ }^{14}$. The negative polarity azimuthal magnetic field pulse indicates that the source is a downward current, reflecting either downward-moving positive charge or upward-moving negative charge, in agreement with previous reports for gigantic jets ${ }^{9}$ and also lightning modelling and observations ${ }^{4}$. The precise time alignment of this signature with the gigantic jet optical emissions (see Fig. 3) and the unusual timescales of the ULF pulse strongly suggest that this pulse originates in current flowing in the jet itself and not in a conventional lightning process. This is consistent with satellite-based optical measurements of gigantic jets that reveal far dimmer optical emissions from the clouds than for typical large lightning flashes ${ }^{11}$.

Additional insight is provided by the ULF data around the onset (Fig. 2b) and a $110 \mathrm{~ms}$ window of triggered VLF data that begins just before the optical jet (Fig. 2c). The slow ULF signal increase before $t=200 \mathrm{~ms}$ begins between $t=-50 \mathrm{~ms}$ and $t=0 \mathrm{~ms}$. There are four distinct ULF pulses in this window, and the first was large enough to trigger our VLF system. All of these originate from downward motion of positive charge, and direction finding and signal dispersion analysis indicate that they originate within $50 \mathrm{~km}$ of the optically estimated gigantic jet location.

The corresponding VLF data show clear signals for the first three ULF pulses. The first is comparable in magnitude to $\sim 24 \mathrm{kA}$ NLDN-detected cloud-to-ground strokes originating in the same region. The ULF data indicate that the slow ramp may have begun around the time of the first pulse, close to $t=-50 \mathrm{~ms}$. One physical situation consistent with the data is that the slow ULF ramp is produced by the upward motion of a negative leader, and the four faster pulses are in-cloud discharges that occurred as the leader experienced instability. The $\sim 50 \mathrm{~ms}$ time window in which the four fast pulses occurred is consistent with a leader travelling at $100 \mathrm{~km} \mathrm{~s}^{-1}$ upward through $5 \mathrm{~km}$ of clouds, after which the leader 


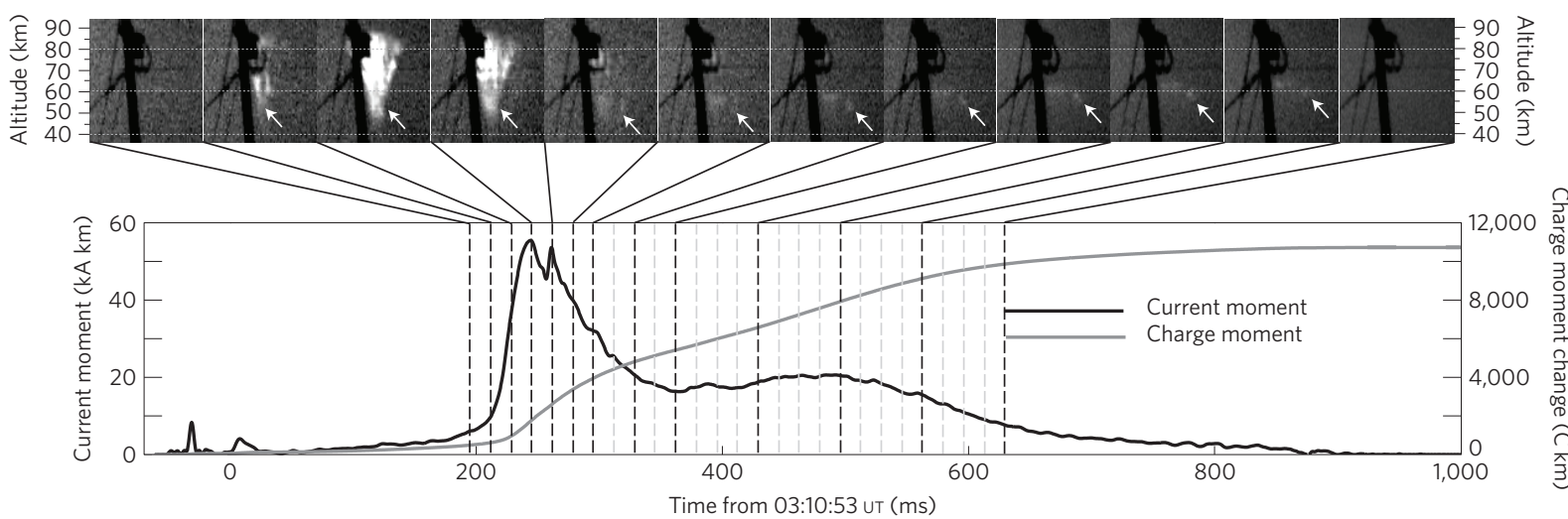

Figure 3 | Time-aligned video fields and the source waveform of the gigantic jet. The dimmer trailing jet feature is marked in the images by the white arrows. The images taken later are averages of two or four individual video fields as noted by the time window on each. The source waveforms shown are the total vertical current moment and the time-integrated vertical charge moment change.

continued to propagate upward above the clouds through clear air. No additional sferics triggered our VLF system for the remainder of the event, indicating that no in-cloud or cloud-to-ground strokes with peak currents greater than approximately $12 \mathrm{kA}$ occurred. These data provide further evidence that the gigantic jet is not linked to any cloud-to-ground lightning stroke $e^{4,11}$ and that the observed slow charge transfer occurs between the cloud and the ionosphere.

Figure 3 shows the relationship between the optical emissions and the instantaneous current moment extracted ${ }^{15}$. The steadily increasing current moment, from $t=0$ to $200 \mathrm{~ms}$, occurs before optical emissions are observed. This is probably the upwardpropagation of the negative leader of the gigantic jet, which can sometimes be seen from the ground $d^{5,9}$, but in our case was probably obscured by the high background brightness.

Figure 3 also shows the integration window of each individual $16.7 \mathrm{~ms}$ video field of the gigantic jet aligned in time with the ULF-measured current moment waveform. The first field with detectable optical emissions shows them extending to the top of the gigantic jet, and corresponds to the sudden rise in current moment at $t=210 \mathrm{~ms}$ that occurred when the streamers reached the ionosphere. The rise time of the main current moment pulse is about $30 \mathrm{~ms}$, and the brightest two images correspond to the periods of highest average current moment and the fully developed jet stage identified by $\mathrm{Su}$ and colleagues? .

At $t=260 \mathrm{~ms}$ the jet brightness drops significantly, and there is a short $(\sim 10 \mathrm{~ms})$ and small ULF pulse at this time that originates from the azimuth of the jet and may be an in-cloud or jet process. The highest altitude optical emissions fade below the background by $t=400 \mathrm{~ms}$ but the lower altitude emissions persist and travel slowly upwards as a trailing jet ${ }^{9}$. During the upward trailing jet propagation, the overall current moment in the jet persists at a nearly constant $20 \mathrm{kA} \mathrm{km}$ (with slow increases and decreases along the way) until $t=550 \mathrm{~ms}$. After this point the optical emissions fall below the background and the total current moment steadily drops below the noise level (about $1 \mathrm{kA} \mathrm{km}$ ) by $t=900 \mathrm{~ms}$.

These measurements show that gigantic jets are capable of transferring substantial charge to the lower ionosphere and are essentially upward lightning from thunderclouds ${ }^{4}$ that delivers charge to the end point of the discharge, like conventional cloudto-ground lightning. Figure 3 shows that the maximum vertical current moment (total current integrated along the channel length) is $55 \mathrm{kA} \mathrm{km}$, equal to a current of $730 \mathrm{~A}$ if we assume a channel length of $75 \mathrm{~km}$. This is much smaller than the short duration current of the return stroke in conventional lightning but is comparable in magnitude and duration to the long continuing current process in conventional lightning ${ }^{16}$, albeit over a channel ten times longer. This similarity suggests that the in-cloud processes during a gigantic jet event may be similar to those during protracted continuing current.

The charge moment change of the jet integrated over its duration of nearly one second is $10,800 \mathrm{C} \mathrm{km}$, corresponding to a total charge transfer from the thunderstorm to the ionosphere of $144 \mathrm{C}$ for the assumed channel length of $75 \mathrm{~km}$. This too is comparable in magnitude to the total charge transfer in large lightning strokes that contain significant continuing currents ${ }^{17,18}$. Given that the charge reservoirs in thunderstorms are the ultimate charge sources in gigantic jets and cloud-to-ground lightning, and both phenomena involve electrical contact between the thunderstorm charge region and a very large conducting surface, it is perhaps not surprising that their charge transfers are comparable.

Previous studies have reported a lack of identifiable electromagnetic signatures of gigantic jets in one out of five events in one case ${ }^{9}$ and in the single event studied in another case ${ }^{10}$. Although both of those previous studies had problems with timing ambiguities that may have made it difficult to identify smaller electromagnetic signatures, the total charge moment change in this jet still exceeds those previously reported ${ }^{9}$ by a factor of $5-10$. This suggests that, like lightning, gigantic jets can span a wide range of total charge transfer, and the event reported here seems to have been a very big one.

\section{Methods}

Video and analysis. The video stills shown in Fig. 3 are from a video recorded at a field site near Duke University $\left(35.975^{\circ} \mathrm{N}, 79.100^{\circ} \mathrm{W}\right)$ using a WATEC $902 \mathrm{H} 2$ Ultimate low light CCD (charge coupled device) camera coupled to a triggered video acquisition system that records approximately one second of video when specified trigger criteria are met. Absolute timing accuracy better than $1 \mathrm{~ms}$ was provided by a GPS-synchronized (GPS: Global Positioning System) time code inserter. The images are extracted from deinterlaced video and are thus integrated over $16.7 \mathrm{~ms}$ time windows.

Horizon features provide a precise azimuth to the jet (note the power pole partly blocking the event) of $137^{\circ}$ east of geographic north. Its range is estimated to be $350 \mathrm{~km}$ by comparison with a previously observed sprite that had a nearly identical vertical extent and whose distance was known from NLDN data. Assuming this range, a star field analysis reveals a maximum jet altitude of $88 \mathrm{~km}$, in agreement with previous jet observations and thus validating the range estimate.

Magnetic measurements and analysis. At the same field site, radio emissions in the vector horizontal magnetic field are measured with two pairs of magnetic induction coils that cover $50 \mathrm{~Hz}-30 \mathrm{kHz}$ (VLF) and $<0.1-400 \mathrm{~Hz}$ (ULF). The signals from the ULF coils are sampled continuously at $2.5 \mathrm{kHz}$ whereas those from the VLF coils are sampled at $100 \mathrm{kHz}$ in $110 \mathrm{~ms}$ windows when the signal magnitude exceeds a prescribed trigger level. For lightning at $350 \mathrm{~km}$ range, measurements show that the trigger level used is equivalent to the signal produced by a $12 \mathrm{kA}$ peak current lightning stroke.

Measurements of the electromagnetic fields produced by lightning are an important tool for remotely measuring lightning properties. For example, lightning strokes can be identified and peak currents can be inferred from distant fields from 1 to $400 \mathrm{kHz}$ (ref. 12). Much slower lightning continuing currents can be detected and measured from distant quasi-static magnetic fields down to a few hertz ${ }^{15,19}$. 
Extracting the source current moment waveform (the integral of the current along the entire channel) that produced a measured ULF magnetic field waveform is relatively straightforward for nearby low-frequency fields ${ }^{15}$, and we do so here using the approach used in ref. 18.

We also roughly estimate the propagation distance of the VLF-observed fast waveforms by comparing them with forward simulations of signals propagating under a typical night-time ionosphere. These signals are significantly dispersed from propagation in the Earth-ionosphere waveguide, and the multiple reflections and other waveform details are strongly dependent on the propagation distance. The VLF signals reported here are consistent with a $350 \mathrm{~km}$ propagation distance but with a substantial $\pm 50 \mathrm{~km}$ uncertainty. This shows that they did originate in the general region of the gigantic jet and may have originated from that precise location.

Received 28 April 2009; accepted 21 July 2009; published online 23 August 2009

\section{References}

1. Pasko, V. P. \& George, J. J. Three-dimensional modeling of blue jets and blue starters. J. Geophys. Res. 107, 1458 (2002).

2. Petrov, N. I. \& Petrova, G. N. Physical mechanisms for the development of lightning discharges between a thundercloud and the ionosphere. Tech. Phys. 44, 472-475 (1999).

3. Sukhorukov, A. I. \& Stubbe, P. Problems of blue jet theories. J. Atmos. Solar Terr. Phys. 60, 725-732 (1998).

4. Krehbiel, P. R. et al. Upward electrical discharges from thunderstorms. Nature Geosci. 1, 233-237 (2008)

5. Pasko, V. P., Stanley, M. A., Mathews, J. D., Inan, U. S. \& Wood, T. G. Electrical discharge from a thundercloud top to the lower ionosphere. Nature 416, 152-154 (2002).

6. Wescott, E. M., Sentman, D. D., Osborne, D. L., Hampton, D. L. \& Heavner, M. J. Preliminary results from the SPRITES94 aircraft campaign 2. Blue jets. Geophys. Res. Lett. 22, 1209-1212 (1995).

7. Lyons, W. A., Nelson, T. E., Armstrong, R. A., Pasko, V. P. \& Stanley, M. A. Upward electrical discharges from thunderstorm tops. Bull. Am. Meteorol. Soc 84, 445-454 (2003)

8. Wescott, E. M. et al. Blue starters: Brief upward discharges from an intense Arkansas thunderstorm. Geophys. Res. Lett. 23, 2153-2156 (1996).

9. $\mathrm{Su}, \mathrm{H}$. T. et al. Gigantic jets between a thundercloud and the ionosphere. Nature 423, 974-976 (2003).
10. van der Velde, O. A. et al. Analysis of the first gigantic jet recorded over continental North America. J. Geophys. Res. 112, D20104 (2007).

11. Kuo, C.-L. et al. Discharge processes, electric field and electron energy in ISUAL-recorded gigantic jets. J. Geophys. Res. 114, A04314 (2009).

12. Cummins, K. L. et al. A combined TOA/MDF technology upgrade of the US National Lightning Detection Network. J. Geophys. Res. 103, 9035-9044 (1998).

13. Cummer, S. A. Current moment in sprite-producing lightning. J. Atmos. Solar Terr. Phys. 65, 499-508 (2003).

14. Greifinger, C. \& Greifinger, P. Approximate method for determining ELF eigenvalues in the Earth-ionosphere waveguide. Radio Sci. 13, 831-837 (1978).

15. Cummer, S. A. \& Füllekrug, M. Unusually intense continuing current in lightning causes delayed mesospheric breakdown. Geophys. Res. Lett. 28, 495-498 (2001)

16. Rakov, V. A. \& Uman, M. A. Lightning (Cambridge Univ. Press, 2003).

17. Berger, K., Anderson, R. B. \& Kroninger, H. Parameters of lightning flashes. Electra 80, 223-237 (1975).

18. Li, J., Cummer, S. A., Lyons, W. A. \& Nelson, T. E. Coordinated analysis of delayed sprites with high speed images and remote electromagnetic fields. J. Geophys. Res. 113, D20206 (2008).

19. Ross, M., Cummer, S. A., Nielsen, T. K. \& Zhang, Y. Simultaneous remote electric and magnetic field measurements of lightning continuing currents. J. Geophys. Res. 113, D20125 (2008)

\section{Acknowledgements}

This work was supported by the Physical and Dynamic Meteorology and Aeronomy Programs of the National Science Foundation.

\section{Author contributions}

S.A.C. drafted the manuscript and supervised the project. J.L. analysed the gigantic jet images. S.A.C., J.L., F.H. and G.L. analysed the electromagnetic signals. N.J. developed and operated the field instruments. W.A.L. and T.E.N. analysed the radar reflectivity and infrared images. All authors contributed to the discussion of the results and the preparation of the manuscript.

\section{Additional information}

Reprints and permissions information is available online at http://npg.nature.com/ reprintsandpermissions. Correspondence and requests for materials should be addressed to S.A.C. 